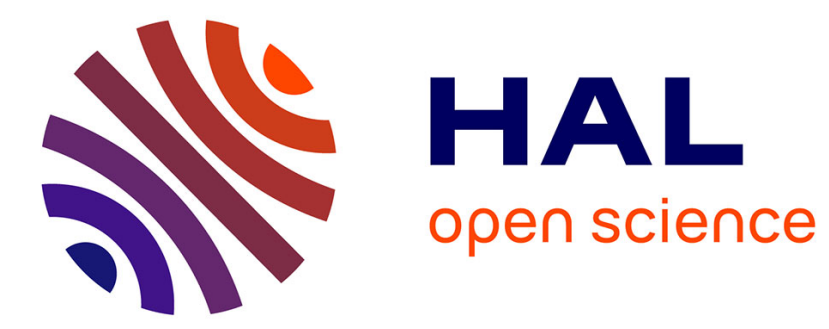

\title{
Social choice and the indexing dilemma
}

Marc Fleurbaey

\section{To cite this version:}

Marc Fleurbaey. Social choice and the indexing dilemma. 2006. halshs-00121371

\section{HAL Id: halshs-00121371 https://shs.hal.science/halshs-00121371}

Preprint submitted on 20 Dec 2006

HAL is a multi-disciplinary open access archive for the deposit and dissemination of scientific research documents, whether they are published or not. The documents may come from teaching and research institutions in France or abroad, or from public or private research centers.
L'archive ouverte pluridisciplinaire HAL, est destinée au dépôt et à la diffusion de documents scientifiques de niveau recherche, publiés ou non, émanant des établissements d'enseignement et de recherche français ou étrangers, des laboratoires publics ou privés. 


\title{
Social choice and the indexing dilemma*
}

\author{
Marc Fleurbaey ${ }^{\dagger}$
}

March 2006

\begin{abstract}
This paper distinguishes an index ordering and a social ordering function as a simple way to formalize the indexing problem in the social choice framework. Two main conclusions are derived. First, the alleged dilemma between welfarism and perfectionnism is shown to involve a third possibility, exemplified by the fairness approach to social choice. Second, the idea that an individual is better off than another whenever he has more (goods, functionings...) in all dimensions, which is known to enter in conflict with the Pareto principle, can be partly salvaged by adopting the fairness approach.
\end{abstract}

\section{Introduction}

All theories of justice involve interpersonal comparisons and have a difficulty in precisely defining how such comparisons must be performed. This is especially true for non-welfarist theories, such as Rawls's in which individual situations are measured in terms of primary goods. Here is an important passage in which Rawls describes the problem, identifies some simplifications, and suggests a solution.

"One problem clearly is the construction of the index of primary social goods. Assuming that the two principles of justice are serially

*This paper has benefited from stimulating interactions with K. Basu, P. Pattanaik, B. Tungodden and the participants at the Philosophical Aspects of Social Choice conference in Caen.

${ }^{\dagger}$ CNRS-CERSES, University of Paris 5, IDEP. Email: marc.fleurbaey@univ-paris5.fr. 
ordered, this problem is greatly simplified. The basic liberties are always equal, and there is fair equality opportunity; one does not need to balance these liberties and rights against other values. The primary social goods that vary in their distribution are the rights and prerogatives of authority, and income and wealth. But the difficulties are not so great as they might seem at first because of the nature of the difference principle. The only index problem that concerns us is that for the least advantaged group. The primary goods enjoyed by other representative individuals are adjusted to raise this index, subject of course to the usual constraints. It is unnecessary to define weights for the more favored positions in any detail, as long as we are sure that they are more favored. But often this is easy since they frequently have more of each primary good that is distributed unequally. If we know how the distribution of goods to the more favored affects the expectations of the most disfavored, this is sufficient. The index problem largely reduces, then, to that of weighting primary goods for the least advantaged. We try to do this by taking up the standpoint of the representative individual from this group and asking which combination of primary social goods it would be rational for him to prefer. In doing this we admittedly rely upon intuitive estimates. But this cannot be avoided entirely." (Rawls 1999, p. 80)

The first simplification mentioned by Rawls is that some dimensions (basic liberties, fair opportunities) have absolute priority and the corresponding goods must be equally distributed. The second simplification, which will be the focus of the second part of this paper, is that the better-off usually have more of each primary good. It then remains to weight the primary goods for the worse-off only, and for this Rawls suggests to rely on "representative" preferences of this subgroup of the population. This suggestion is however vague and seems to involve a kind of averaging.

In a famous comment, Arrow expressed scepticism about this suggestion.

"So long as there is more than one primary good, there is an indexnumber problem in commensurating the different goods, which is in principle as difficult as the problem of interpersonal comparability with which we started" (Arrow 1973, p. 254).

An exchange between Kolm and Rawls on this issue is illuminating. Kolm (1972) proposed to tackle the comparison problem by relying on "fundamen- 
tal preferences", that is, preferences over personal characteristics in addition to external goods. Rawls firmly rejected this idea.

"The notion of a shared highest-order preference function is plainly incompatible with the conception of a well-ordered society in justice as fairness. For in the circumstances of justice citizen's conceptions of the good are not only said to be opposed but to be incommensurable." (Rawls 1982, p. 179) "To an economist (...) an index of primary goods may seem merely ad hoc patchwork not amenable to theory. (...) The economist's reaction is partly right: an index of primary goods does not belong to theory in the economist's sense. It belongs instead to a conception of justice which falls under the liberal alternative to the tradition of the one rational good. Thus the problem is not how to specify an accurate measure of some psychological or other attribute available only to science. Rather, it is a moral and practical problem." (pp. 184-185)

This explanation is quite interesting. Rawls rejects the idea that interpersonal comparisons of bundles of primary goods could be based on a theoretical notion of utility or well-being, and makes it clear that the index must instead reflect ethical priorities in the practical problem of sharing resources. In other words, fairness considerations should somehow determine the construction of the index. ${ }^{1}$

This is however insufficient to address Arrow's challenge. Arneson (1990) adds a dramatic flavor to this issue, in the form of a dilemma. If, as suggested by Rawls himself, the index assigns the same weights to the various primary goods in all individual bundles, independently of the diverse personal preferences, then one can consider this imposition of external weights as a kind of perfectionnism. Assuming that the weights are faithful to the preferences of a "representative" individual of the worse-off group, those with non-representative preferences may indeed complain that the evaluation of their personal situation is submitted to someone else's conception of the good

\footnotetext{
${ }^{1}$ Robbins (1932) defended the more radical thesis that the measurement of interpersonally comparable utility itself is necessarily plagued with value judgments, without however precisely describing the substance of such value judgments. Here Rawls embraces the idea that (a particular kind of) value judgments must shape the index. One also finds the related idea that fairness principles would make it possible to "pair" the indifference curves of different individuals in Bergson (1954).
} 
life. The alternative is to make the index depend precisely on everyone's preferences, but then Rawls's resourcist approach seems bound to dissolve into a kind of welfarism.

This alleged dilemma is the main topic of this paper. In a remarkable comment, Roemer objects to it.

"Arneson is not right to conclude that the Rawlsian view must dissolve into welfarism -in particular, into equalizing (or maximinning) welfare. There may be room for a theory which chooses indices of primary goods which are ordinally equivalent to welfare... Such a theory would not be welfarist, as these indices need not be recoverable from information on welfare levels. The task for a Rawlsian must be to find such indices which are justifiable without appeal to a perfectionist standard or to the inherent superiority of some life plans over others." (Roemer 1996, pp. 171-172)

Roemer does not substantiate his claim by an example or by precise suggestions about how to proceed, but this paper will definitely concur with him in pointing at a third possibility and will flesh it out in a more precise way.

The first part of this paper analyzes this dilemma. It introduces a simple formal framework in which an "index ordering" ranks individual situations, each of which is described by a bundle of goods (or, more generally, functionings) and by an individual utility function. The welfarist approach evaluates such situations by the level of utility obtained at the contemplated bundle, whereas a perfectionnist ordering typically disregards individual preferences and directly focuses on bundles. We will show how the welfarismperfectionnism dilemma relates to Arrow's theorem of social choice and identify a third possibility, the "fairness approach", that represents an interesting way out of the dilemma. The main ideas here are not new, and can be found for instance in Fleurbaey and Hammond (2004) and Fleurbaey (2003). But the formal framework introduced here is quite helpful in making the analysis of the indexing problem more transparent, by contrast with the standard social choice approach which focuses on the social aggregation problem and therefore on the construction of a social ordering function rather than an index of interpersonal comparisons.

The second part of the paper examines the "dominance" principle according to which one should always consider that an individual having more than 
another is unambiguously better-off. This very natural idea, mentioned by Rawls in the first quotation above, has been eloquently developed by Sen.

"The existence of different ends does not, of course, rule out the possibility that different people may have exactly the same ranking of the different means in the form of bundles of primary goods... The problem of indexing of primary goods bundles would be considerably simplified if the orderings of these means corresponding to the respective ends had extensive overlaps. The larger the "intersection", the greater the possibility of using an agreed index of primary goods bundles." (Sen 1991, p. 16) ${ }^{2}$

A particular case of agreement between individuals, when they have monotonic preferences, is that a greater bundle is better than a smaller one.

"Having more of each relevant functioning or capability is a clear improvement, and this is decidable without waiting to get agreement on the relative weights to be attached to the different functionings and capabilities... The 'intersection approach', which articulates only those judgements that are shared implications of all of the possible alternative weights, can indeed take us quite a distance" (Sen 1992, pp. 46-47).

Commenting on Rawls, Sen or other sources, various authors (Gibbard 1979, Basu 1994, Fleurbaey and Trannoy 2003, Brun and Tungodden 2004, Pattanaik and $\mathrm{Xu} 2005$ ) have, however, questioned this intuitive idea and noticed that it can easily lead to a contradiction with the Pareto principle. In this paper we will rely on the formalism of the index ordering in order to analyze the problem. Eventually we will relate this dominance-Pareto dilemma to the welfarism-perfectionnism dilemma studied in the first part of the paper, and show that the third way identified for the latter, the so-called fairness approach, can be useful in the search of a reasonable compromise for the former.

\footnotetext{
${ }^{2}$ Rawls (1993) comments on this suggestion in quite favorable terms. "We suppose that all citizens have a rational plan of life that requires for its fulfillment roughly the same kind of primary goods... Without assumptions of this kind, the index problem is known to be insoluble." (p. 181)
} 


\section{The model}

The formalism is reduced to the minimum necessary for the analysis. The population is made of $n \geq 2$ individuals, and is denoted $N=\{1, \ldots, n\}$. Every individual's consumption set is $X=\mathbb{R}_{+}^{\ell}$, and the $\ell \geq 2$ dimensions may be interpreted as referring to goods (primary goods or ordinary commodities) or to functionings more generally. Let $X^{*}=\mathbb{R}_{+}^{\ell} \backslash\{0\}$. Every individual $i$ has a utility function $U_{i}$ on $X$, which is an ordinary utility function measuring happiness or preference satisfaction. The important point here that this utility function is not necessarily defining the ethical index by which individual situations are compared. We assume however that this utility function represents the individual's preference ordering (an ordering is a complete and transitive binary relation) that the Pareto principle is meant to sanctify. Let $\mathcal{U}$ be the set of utility functions on $X$ that are continuous, strictly monotonic, and convex.

A profile of utility functions is denoted $U=\left(U_{1}, \ldots, U_{n}\right)$ and an allocation is denoted $x=\left(x_{1}, \ldots, x_{n}\right)$, where $x_{i} \in X$ denotes individual $i$ 's personal bundle.

The social choice problem is to define a social ordering function (SOF) from $\mathcal{U}^{n}$ to the set of orderings on $X^{n}$, which for every profile $U$ determines an ordering $\bar{R}(U)$ that ranks allocations. The notation $x \bar{R}(U) y$ will mean that $x$ is weakly better than $y$. Let $\bar{P}(U)$ and $\bar{I}(U)$ denote the strict preference and indifference counterparts of the ordering, respectively.

The index problem is to define an index ordering $\succcurlyeq$ applying to individual situations $(a, u)$ where $a$ is a bundle from $X$ and $u$ a utility function from $\mathcal{U}$. Let $\left(x_{i}, U_{i}\right) \succcurlyeq\left(x_{j}, U_{j}\right)$ mean that $i$ is at least as well-off than $j$, in their respective situations, and let $\succ, \sim$ stand for "strictly better-off" and "equally well-off", respectively.

In this simple model, utility functions and bundles in $X$ are the only possible differences between individuals (apart from their labels $1, \ldots, n$ ). One might imagine a richer structure in which, for instance, personal resources (or characteristics) would be distinguished from external resources. This is not needed for the purpose of this analysis.

This simple way of formalizing the index problem can be compared to related approaches in the literature. The special theory of social choice that focuses the informational basis of interpersonal comparisons standardly assumes that the index problem has been solved and focuses on the social aggregation problem. In the most common framework, introduced in Sen 
(1970) and developed later by him and many authors, it assumes that individual utility functions, or families thereof, do embody the ethical indexes on which comparisons can be made. In a different framework, also developed by the same group of authors, there is an "extended sympathy" index that compares extended alternatives $(x, i)$ where $x$ is e.g. an allocation and $i$ is an individual. This framework is not so different as such an index can typically be represented by comparable utility functions.

Social choice theory assumes that such methods of interpersonal comparisons are available and examines the possibilities of social aggregation depending on the properties of such indexing methods. In contrast, the framework of this paper enables us to study the construction of the index, by imposing some ethical requirements on this index itself, and also possibly on the social aggregation that might rely on the index. In particular, it leaves it open whether the ethical index should rely on utility values or not.

\section{The social choice approach}

We first rehearse particular versions of the fundamental results of social choice, for this framework, in order to set the stage for the rest of the analysis.

The following requirements imposed on the SOF $\bar{R}$ do not directly refer to the indexing problem, but can indirectly be interpreted in these terms. Pareto axioms convey the idea that for any given individual, his own preferences should determine how his situation is evaluated. The independence axiom means that utility levels at a particular situation contain the relevant information, at the exclusion of utility levels at other alternatives. The ordinality axiom adds that only ordinal preferences should be taken into account, at the exclusion of utility numbers. These two axioms together form a logical decomposition of Arrow's independence axiom according to which the ranking of two alternatives should only depend on how they are ranked in individual preference orderings. ${ }^{3}$

Weak Pareto: $\forall x, y \in X^{n}, \forall U \in \mathcal{U}^{n}$, if $\forall i \in N, U_{i}\left(x_{i}\right)>U_{i}\left(y_{i}\right)$, then $x \bar{P}(U) y$.

\footnotetext{
${ }^{3}$ See Fleurbaey and Hammond (2003) or Fleurbaey and Mongin (2005) for a detailed discussion of this point.
} 
Pareto Indifference: $\forall x, y \in X^{n}, \forall U \in \mathcal{U}^{n}$, if $\forall i \in N, U_{i}\left(x_{i}\right)=U_{i}\left(y_{i}\right)$, then $x \bar{I}(U) y$.

Independence of Irrelevant Utilities: $\forall x, y \in X^{n}, \forall U, U^{\prime} \in \mathcal{U}^{n}$, if $\forall i \in N, U_{i}\left(x_{i}\right)=U_{i}^{\prime}\left(x_{i}\right)$ and $U_{i}\left(y_{i}\right)=U_{i}^{\prime}\left(y_{i}\right)$, then $x \bar{R}(U) y \Leftrightarrow x \bar{R}\left(U^{\prime}\right) y$.

Ordinal Non-Comparability: $\forall U, U^{\prime} \in \mathcal{U}^{n}$,

if $\forall i \in N, U_{i}$ and $U_{i}^{\prime}$ are ordinally equivalent, then $\bar{R}(U)=\bar{R}\left(U^{\prime}\right)$.

The proof of the two theorems below can be found, for this setting, in Bordes and Le Breton (1989). The first is a version of Arrow's theorem. The second replaces Weak Pareto by Pareto Indifference, and is a direct corollary of Wilson's theorem.

Theorem 1 If $\bar{R}$ satisfies Ordinal Non-Comparability, Weak Pareto and Independence of Irrelevant Utilities, then there exists $i_{0} \in N$ such that: $\forall x, y \in\left(X^{*}\right)^{n}, \forall R \in D$, if $x_{i_{0}} P_{i_{0}} y_{i_{0}}$, then $x \bar{P}(R) y$.

Theorem 2 If $R$ satisfies Ordinal Non-Comparability, Pareto Indifference and Independence of Irrelevant Utilities, then one of the three following assertions is true:

(i) $\forall x, y \in\left(X^{*}\right)^{n}, \forall R \in D, x \bar{I}(R) y$;

(ii) $\forall x, y \in\left(X^{*}\right)^{n}, \forall R \in D$, if $x_{i_{0}} P_{i_{0}} y_{i_{0}}$, then $x \bar{P}(R) y$;

(iii) $\forall x, y \in\left(X^{*}\right)^{n}, \forall R \in D$, if $x_{i_{0}} P_{i_{0}} y_{i_{0}}$, then $y \bar{P}(R) x$.

\section{A simple formulation of the indexing prob- lem}

As mentioned in the previous section, the standard axioms of social choice bear on the social aggregation problem and only indirectly impose conditions on interpersonal comparisons. We now introduce axioms that bear directly on the index ordering. As it turns out, the Pareto, independence and ordinality conditions have immediate translations in this setting.

The Pareto axioms below state that the evaluation of individual situations should respect individual preferences, without implying anything about social evaluations. 
$\sim$ Weak Pareto: $\forall a, b \in X, \forall u \in \mathcal{U}$, if $u(a)>u(b)$, then $(a, u) \succ(b, u)$.

$\sim$ Pareto Indifference: $\forall a, b \in X, \forall u \in \mathcal{U}$, if $u(a)=u(b)$, then $(a, u) \sim(b, u)$.

For later reference, it is also useful to introduce a strong Pareto axiom.

$\sim$ Strong Pareto: $\forall a, b \in X, \forall u \in \mathcal{U}$, $(a, u) \succcurlyeq(b, u)$ if and only if $u(a) \geq u(b)$.

The independence axiom now strictly says that the evaluation of individual situations should be based on utility levels at the contemplated situations and not on other utility levels.

Independence of Irrelevant Utilities: $\forall a, b \in X, \forall u, u^{\prime}, v, v^{\prime} \in \mathcal{U}$, if $u(a)=u^{\prime}(a)$ and $v(b)=v^{\prime}(b)$, then $(a, u) \succcurlyeq(b, v) \Leftrightarrow\left(a, u^{\prime}\right) \succcurlyeq\left(b, v^{\prime}\right)$.

Similarly, the ordinality condition now strictly says that preference orderings only should guide interpersonal comparisons of situations.

$\sim$ Ordinal Non-Comparability: $\forall a, b \in X, \forall u, u^{\prime}, v, v^{\prime} \in \mathcal{U}$,

if $u$ and $u^{\prime}$ (resp. $v$ and $v^{\prime}$ ) are ordinally equivalent, then $(a, u) \succcurlyeq(b, v) \Leftrightarrow\left(a, u^{\prime}\right) \succcurlyeq\left(b, v^{\prime}\right)$.

It may be useful to note that the independence and ordinality axioms have two equivalent and somewhat simpler formulations, as described in the following lemma.

Lemma $1 \sim$ Independence of Irrelevant Utilities is equivalent to: $\forall a \in X$, $\forall u, u^{\prime} \in \mathcal{U}$, if $u(a)=u^{\prime}(a)$, then $(a, u) \sim\left(a, u^{\prime}\right)$.

$\sim$ Ordinal Non-Comparability is equivalent to: $\forall a \in X, \forall u, u^{\prime} \in \mathcal{U}$, if $u$ and $u^{\prime}$ are ordinally equivalent, then $(a, u) \sim\left(a, u^{\prime}\right)$.

Proof. Sufficiency is obvious, and we focus on necessity. Let $\succcurlyeq$ satisfy $\sim$ IIU, and let $a$ and $u, u^{\prime}$ be such that $u(a)=u^{\prime}(a)$. By reflexivity, $(a, u) \sim(a, u)$. By $\sim \mathrm{IIU},(a, u) \sim\left(a, u^{\prime}\right)$.

Let $\succcurlyeq$ satisfy $\sim$ ONC, and let $a$ and $u, u^{\prime}$ be such that $u$ and $u^{\prime}$ are ordinally equivalent. By reflexivity, $(a, u) \sim(a, u)$. By $\sim \mathrm{ONC},(a, u) \sim\left(a, u^{\prime}\right)$.

The main result of this first part of the paper is the following theorem. It says that the combination of Pareto, independence and ordinality conditions at the level of the index ordering is problematic, and leads either to general indifference or to an outright impossibility. 
Theorem 3 If $\succcurlyeq$ satisfies $\sim$ Pareto Indifference, $\sim$ Independence of Irrelevant Utilities and $\sim$ Ordinal Non-Comparability, then: $\forall a, b \in X^{*}, \forall u, v \in \mathcal{U}$, $(a, u) \sim(b, v)$. If $\sim$ Pareto Indifference is replaced by $\sim$ Weak Pareto, then one has an incompatibility.

Proof. First part. Let $a, b$ and $u, u^{\prime}$ be such that $u(a)=u^{\prime}(b)$. Let $c$ and $v$ be such that $u(a)=u(c)=v(c)=v(b)$. Such a $c$ and $v$ exist since $a, b \in X^{*}$. By $\sim$ PI, $(a, u) \sim(c, u)$. By $\sim$ IIU (and Lemma 1), $(c, u) \sim(c, v)$. By $\sim$ PI, $(c, v) \sim(b, v)$. By $\sim$ IIU (and Lemma 1$),(b, v) \sim\left(b, u^{\prime}\right)$. In conclusion, $(a, u) \sim\left(b, u^{\prime}\right)$.

Take any $a, b$ and $u, v$. Let $u^{\prime}, v^{\prime}$ be such that $u$ and $u^{\prime}$ (resp. $v$ and $v^{\prime}$ ) are ordinally equivalent, while $u^{\prime}(a)=v^{\prime}(b)$. By the above fact, $\left(a, u^{\prime}\right) \sim\left(b, v^{\prime}\right)$. By $\sim \mathrm{ONC},(a, u) \sim(b, v)$.

Second part. Let $a, b$ and $u, u^{\prime}$ be such that $u(a)>u^{\prime}(b)$. Let $c$ and $v$ be such that $u(a)>u(c)$ and $u(c)=v(c)>v(b)=u^{\prime}(b)$. By $\sim \mathrm{WP},(a, u) \succ$ $(c, u)$. By $\sim \mathrm{IIU}$ (and Lemma 1), $(c, u) \sim(c, v)$. By $\sim \mathrm{WP},(c, v) \succ(b, v)$. By $\sim$ IIU (and Lemma 1$),(b, v) \sim\left(b, u^{\prime}\right)$. In conclusion, $(a, u) \succ\left(b, u^{\prime}\right)$.

Take any $a, b$ and $u, v$ such that $u(a)>v(b)$. Let $u^{\prime}, v^{\prime}$ be such that $u$ and $u^{\prime}$ (resp. $v$ and $v^{\prime}$ ) are ordinally equivalent, while $u^{\prime}(a)<v^{\prime}(b)$. By the above fact, $(a, u) \succ(b, v)$ and $\left(a, u^{\prime}\right) \prec\left(b, v^{\prime}\right)$. By $\sim \mathrm{ONC}$, this is impossible.

Notice that the proof is incomparably simpler than the proof of the social choice theorems. It contains a neutrality step that is reminiscent of the welfarism lemma one encounters in social choice (see d'Aspremont and Gevers 1977, d'Aspremont 1985).

\section{Back to social choice}

Let us now see how the theorem of the previous section relates to the social choice theorems. We focus on the second social choice theorem (that involving Pareto indifference), for which the connection is more interesting, and leave it to the reader to examine the first theorem. One can connect the two kinds of results by means of the following axiom, which requires the SOF $\bar{R}$ to rely on the index ordering $\succcurlyeq .4$

Pareto Indifference wrt $\succeq: \forall x, y \in X^{n}, \forall U \in \mathcal{U}^{n}$,

if $\forall i \in N,\left(x_{i}, U_{i}\right) \sim\left(y_{i}, U_{i}\right)$, then $x \bar{I}(U) y$.

\footnotetext{
${ }^{4}$ This axiom is reminiscent of the principle of personal good in Broome (1991).
} 
The three axioms involved in the second social choice theorem can be derived from this axiom and the conditions imposed on the index ordering, as detailed in the following proposition, whose immediate proof is omitted.

Proposition 1 If $\bar{R}$ satisfies Pareto Indifference wrt an index that satisfies $\sim$ Pareto Indifference (resp., $\sim$ Independence of Irrelevant Utilities, $\sim$ Ordinal Non-Comparability), then it satisfies Pareto Indifference, (resp., Independence of Irrelevant Utilities, Ordinal Non-Comparability).

This proposition shows that requiring the SOF to satisfy Pareto Indifference wrt an index $\succcurlyeq$ that satisfies $\sim$ Pareto Indifference, $\sim$ Independence of Irrelevant Utilities and $\sim$ Ordinal Non-Comparability is at least as demanding as requiring it to satisfy Pareto Indifference, Independence of Irrelevant Utilities and Ordinal Non-Comparability (as in the second social choice theorem). We will see that it is actually more demanding. There is only a partial converse to this result.

Proposition 2 If $\bar{R}$ satisfies Pareto Indifference and Independence of Irrelevant Utilities, then it satisfies Pareto Indifference wrt an index that satisfies $\sim$ Pareto Indifference and $\sim$ Independence of Irrelevant Utilities.

The index ordering involved in the (immediate) proof of this proposition is the welfarist ordering $\succcurlyeq_{w}$ defined by $(a, u) \succcurlyeq_{w}(b, v) \Leftrightarrow u(a) \geq v(b)$. This index ordering does not satisfy $\sim$ Ordinal Non-Comparability.

The following result is a direct corollary of the theorem of the previous section, and is an adaptation of the second social choice theorem in our framework. The dictatorship and anti-dictatorship parts of the latter have disappeared, which proves that this new version of the social choice result involves stronger requirements.

Corollary 1 If $\bar{R}$ satisfies Pareto Indifference wrt an index $\succcurlyeq$ that satisfies $\sim$ Pareto Indifference, $\sim$ Independence of Irrelevant Utilities and $\sim$ Ordinal Non-Comparability, then: $\forall x, y \in X^{n}, \forall R \in D, x \bar{I}(R) y$.

Even though this last result tells us nothing really new about the social choice problem, it may be worthwhile to have a result which clearly separates the conditions bearing on the social aggregation problem from the conditions bearing on the index problem. Moreover, with this slightly more demanding set of requirements (as compared to the second social choice theorem) the proof of the result is considerably simplified. 


\section{A welfarism-perfectionnism dilemma?}

Theorem 3 involves three axioms and implies an unappealing index ordering that is totally unable to discriminate individual situations. Let us examine the new possibilities that emerge when one of the axioms is relaxed.

Let us first drop $\sim$ Pareto Indifference. The two other axioms are satisfied by any index $\succcurlyeq$ that is independent of $u$. That is, for all $a \in X$ and $u, u^{\prime} \in \mathcal{U}$, $(a, u) \sim\left(a, u^{\prime}\right)$. We will say that an index $\succcurlyeq$ is perfectionnist if it is satisfies this property of being impervious to the concerned individual's preferences. ${ }^{5}$

Let us now drop $\sim$ Ordinal Non-Comparability. Then the welfarist index ordering $\succcurlyeq_{w}$ becomes acceptable. We will say that an index $\succcurlyeq$ is weakly welfarist if $(a, u) \sim\left(b, u^{\prime}\right)$ whenever $(a, u) \sim_{w}\left(b, u^{\prime}\right)$, and that it is welfarist if $\succcurlyeq=\succcurlyeq_{w}$.

Finally, let us consider dropping Independence of Irrelevant Utilities. Then one can find examples of admissible index orderings in the fairness literature. For instance, an egalitarian-equivalent index ordering, inspired by Pazner and Schmeidler (1978) and Pazner (1979), can be defined by selecting a fixed bundle $x_{0}$ and by letting

$$
(a, u) \succcurlyeq(b, v) \Longleftrightarrow \exists \lambda \geq 0, u(a) \geq u\left(\lambda x_{0}\right) \text { and } v\left(\lambda x_{0}\right) \geq v(b) .
$$

This index ordering obviously depends only on ordinal preferences, and it also satisfies $\sim$ Pareto Indifference since when $u(a)=u(b)$, there exists $\lambda$ such that

$$
u(a)=u(b)=u\left(\lambda x_{0}\right),
$$

implying $(a, u) \sim(b, u)$. The fairness literature contains many other examples of similar index orderings. ${ }^{6}$

These three alleys of possibility show that there is no welfarism-perfectionnism dilemma and confirm Roemer's statement that a third way is open. The fairness approach to social choice exemplifies this possibility. There is, however,

\footnotetext{
${ }^{5}$ One might want to reserve the adjective "perfectionnist" to indexes satisfying more substantive properties related to ethical norms. The point of this analysis is just to focus on the property of independence of the index with respect to personal preferences, as in Arneson (1990).

${ }^{6}$ The adoption of the social choice methodology in order to derive social ordering functions on the basis of fairness principles has yielded a variety of SOFs for various settings (see e.g. Fleurbaey and Maniquet 2005, 2006, Fleurbaey 2005). These SOFs all violate Independence of Irrelevant Utilities (and Arrow's independence axiom), and this can be justified by the fact that fairness principles reasonably require using more information than allowed by such independence requirements.
} 
a grain of truth in the alleged dilemma. As stated in the following result, assuming independence it is indeed hard to escape any of the two horns.

Theorem 4 Assume that $\succcurlyeq$ satisfies $\sim$ Independence of Irrelevant Utilities. If it satisfies $\sim$ Pareto Indifference, then it is weakly welfarist. If it satisfies $\sim$ Strong Pareto, then it is welfarist. If it satisfies $\sim$ Ordinal NonComparability, then it is perfectionnist.

Proof. Let $\succcurlyeq$ satisfy $\sim$ IIU and $\sim$ PI. Let $a, b$ and $u, u^{\prime}$ be such that $u(a)=$ $u^{\prime}(b)$. The proof that $(a, u) \sim\left(b, u^{\prime}\right)$ is in the beginning of the proof of Theorem 2.

Let $\succcurlyeq$ satisfy $\sim$ IIU and $\sim$ SP. Let $a, b$ and $u, u^{\prime}$ such that $u(a)>u^{\prime}(b)$. Take $v$ and $c$ such that $u(a)=v(a)>v(c)=u^{\prime}(b)$. By $\sim$ IIU (and Lemma 1), $(a, u) \sim(a, v)$ and by weak welfarism, $(c, v) \sim\left(b, u^{\prime}\right)$. By $\sim \mathrm{SP},(a, v) \succ(c, v)$. By transitivity, $(a, u) \succ\left(b, u^{\prime}\right)$.

Let $\succcurlyeq$ satisfy $\sim$ IIU and $\sim$ ONC. Take any $a$ and $u, u^{\prime}$. Pick $v$ such that $v(a)=u^{\prime}(a)$ and $u$ and $v$ are ordinally equivalent. By $\sim \mathrm{ONC}$ (and Lemma 1), $(a, u) \sim(a, v)$. By $\sim \mathrm{IIU}$ (and Lemma 1), $(a, v) \sim\left(a, u^{\prime}\right)$. By transitivity, $(a, u) \sim\left(a, u^{\prime}\right)$

The welfarism-perfectionnism dilemma is therefore real, but only if one wants to retain the independence requirement. Is such a requirement compelling? The fairness approach strongly suggests that it is not. It may be quite relevant, for the comparison of two individual situations $(a, u)$ and $(b, v)$, to know how the individuals rank them with respect to a reference situation involving a bundle $\lambda x_{0}$. For instance, suppose that, according to his own preferences, $i$ is worse-off at $\left(x_{i}, U_{i}\right)$ than at $\left(\Omega / n, U_{i}\right)$, where $\Omega / n$ is the per capita amount of resources to be distributed, and that in contrast $j$ is better-off at $\left(x_{j}, U_{j}\right)$ than at $\left(\Omega / n, U_{j}\right)$. Assuming, as in Steinhaus (1948), that individuals have a right to the per capita amount of resources, there seems to be a case for arguing that $i$ is less well treated than $j$, and is therefore relevantly worse-off. This kind of evaluation is ruled out by Independence of Irrelevant Utilities and this appears quite questionable.

\section{Dominance versus Pareto?}

Let us now examine Rawls's and Sen's suggestion that partial agreement between individuals may alleviate the indexing problem. We will focus on 
the minimal version of this intersection approach, namely, the idea that when one individual has more in all dimensions than another, she is unambiguously better-off.

It has already been shown, by Gibbard (1979), Fleurbaey and Trannoy (2003) and Brun and Tungodden (2004) that, if one relies on this idea in order to formulate transfer axioms applied to a SOF, such a SOF could not simultaneously satisfy Pareto requirements. Such transfer axioms say that when an individual has more of every good than another, then a transfer between them that does not eliminate this dominance configuration is a social improvement. These axioms are unfortunately adding an egalitarian requirement to the dominance idea. Such a requirement, no matter how compelling, is logically independent. Once again, it appears useful to focus on the requirements one wants to impose on the index ordering itself rather than on indirect requirements passing via axioms on SOFs. Let us therefore formulate the dominance principle directly as a property of $\succcurlyeq$. More precisely, we consider the possibility that it applies only on a subset of bundles, $A \subset X$.

Dominance on $A: \forall a, b \in A, \forall u, v \in \mathcal{U}$, if $a \gg b$, then $(a, u) \succ(b, v)$.

Similarly, we reformulate the $\sim$ Weak Pareto condition with the possibility that it applies only to a subset of utility functions, $\mathcal{V} \subset \mathcal{U}$.

$\sim$ Weak Pareto on $\mathcal{V}: \forall a, b \in X, \forall u \in \mathcal{V}$ if $u(a)>u(b)$, then $(a, u) \succ(b, u)$.

We need a few definitions and pieces of notation. A set $A \subset X$ is thin if for all $a, b \in A$, either $a \geq b$ or $a \leq b$. A set $\mathcal{V} \subset \mathcal{U}$ is thin if for all $u, v \in \mathcal{V}$, $u$ and $v$ are ordinally equivalent. If $d$ denotes the Euclidean distance, then $B_{\varepsilon}(a)=\{q \in X \mid d(q, a) \leq \varepsilon\}$.

The following theorem delineates the conflict between Dominance and Pareto. It shows that for the dominance idea to be satisfied by a Paretian index, one has to assume not only partial agreement between individuals, but full agreement over the ranking of all situations (involving bundles in the subset $A$ ). The theorem contains an assumption on $A$ that excludes degenerate cases in which $A$ would contain only a few scattered points.

Theorem 5 Suppose $A$ is such that for all $a \in A, \varepsilon>0$, there is $b \in B_{\varepsilon}(a)$ such that $b \gg a$, and if $a \gg 0$, there is $c \in B_{\varepsilon}(a)$ such that $c \ll a$. 
(i) If $\succcurlyeq$ satisfies $\sim$ Dominance on $A$ and $\sim$ Weak Pareto on $\mathcal{U}$, then $A$ is thin.

(ii) If $\succcurlyeq$ satisfies $\sim$ Dominance on $X$ and $\sim$ Weak Pareto on $\mathcal{V}$, then $\mathcal{V}$ is thin.

(iii) If $\succcurlyeq$ satisfies $\sim$ Dominance on $A$ and $\sim$ Weak Pareto on $\mathcal{V}$, then for all $a, b \in A, u, v \in \mathcal{V}, u(a) \geq u(b)$ if and only $v(a) \geq v(b)$.

Proof. The first two assertions are corollaries of the last one. Suppose that there is $a, b \in A, u, v \in \mathcal{V}$, such that $u(a) \geq u(b)$ and $v(a)<v(b)$. By continuity of the utility functions and the assumption on $A$, one can find $a^{\prime} \in A, a^{\prime} \gg a$ such that $u\left(a^{\prime}\right)>u(b)$ and $v\left(a^{\prime}\right)<v(b)$. By continuity of the utility functions and the assumption on $A$ again, one can find $c, d \in A$ such that $c \ll a^{\prime}, d \gg b$ and $u(c)>u(d)$. By $\sim$ Dominance on $A,(d, u) \succ(b, v)$ and $\left(a^{\prime}, v\right) \succ(c, u)$. By $\sim$ Weak Pareto on $\mathcal{V},(c, u) \succ(d, u)$ and $(b, v) \succ\left(a^{\prime}, v\right)$. One obtains a cycle:

$$
(d, u) \succ(b, v) \succ\left(a^{\prime}, v\right) \succ(c, u) \succ(d, u),
$$

which concludes the proof.

This result questions the intuitive idea that whenever all utility functions agree that a bundle $a$ is better than a bundle $b$, one must have $(a, u) \succ(b, v)$ for any pair of functions $u, v$. If one wants to stick to the Paretian principle of respect of individual preferences, it seems that the dominance principle must be seriously watered down. This can be understood as follows. Even if $x_{i} \gg x_{j}$, for instance, it may happen that agent $i$ is far from her ideal mix of goods, whereas agent $j$ is close to his ideal combination. In such a case, one could easily improve $i$ 's situation by giving her another bundle $y_{i}$ that $j$ would consider to be worse than $x_{j}$. Their different preferences, therefore, make it dubious to compare their situations on the sole basis of physical dominance of bundles.

\section{From dominance to fairness}

In this section we examine the connection between the dominance-Pareto dilemma and the welfarism-perfectionnism-fairness "trilemma".

Let us first observe that the dominance axiom, by implying comparisons that are independent of preferences, has a strong connection with perfectionnism. This can be seen by introducing a continuity condition. 
$\sim$ Continuity: $\forall a \in X, \forall u, v \in \mathcal{U}$, the sets $\{q \in X \mid(q, v) \succcurlyeq(a, u)\}$ and $\{q \in X \mid(a, u) \succcurlyeq(q, v)\}$ are closed.

Proposition 3 If $\succcurlyeq$ satisfies $\sim$ Dominance on $X$ and $\sim$ Continuity, then $\succcurlyeq$ is perfectionnist.

The $\sim$ Continuity axiom would not even be needed if the dominance axiom was formulated in terms of large inequalities:

$$
a \geq b \Rightarrow \forall u, v \in \mathcal{U},(a, u) \succcurlyeq(b, v) .
$$

A reasonable solution, if one wants to stick to the Pareto principle but nonetheless retain as much as possible from the dominance idea, is to restrict the application of the dominance principle to a thin set of bundles, as in assertion (i) of Theorem 5. This avoids perfectionnism, and, in the light of Theorem 3, it remains to see whether one wants to adopt the welfarist or the fairness approach in the definition of the index.

The next theorem says that the fairness approach is actually the only possibility. In particular, the dominance idea, even "thinly" applied, then implies $\sim$ Ordinal Non-Comparability and thereby bars welfarism.

Theorem 6 Suppose $A$ is such that for all $a \in A, \varepsilon>0$, there is $b \in$ $B_{\varepsilon}(a)$ such that $b \gg a$, and if $a \gg 0$, there is $c \in B_{\varepsilon}(a)$ such that $c \ll a$. Suppose moreover that for all $a \in X$, all $u \in \mathcal{U}$, there exists $b \in A$ such that $u(a)=u(b)$. If $\succcurlyeq$ satisfies $\sim$ Dominance on $A, \sim$ Weak Pareto on $\mathcal{U}$ and $\sim$ Continuity, then it also satisfies $\sim$ Ordinal Non-Comparability and violates $\sim$ Independence of Irrelevant Utilities.

Proof. First note that $\sim \mathrm{D}$ and $\sim \mathrm{C}$ imply that for all $a \in A$ and all $u, u^{\prime} \in \mathcal{U}$, $(a, u) \sim\left(a, u^{\prime}\right)$; and that $\sim \mathrm{WP}$ and $\sim \mathrm{C}$ imply $\sim$ Pareto Indifference.

Let $u, u^{\prime} \in \mathcal{U}$ be ordinally equivalent and assume that for some $a \in X$, $(a, u) \nsim\left(a, u^{\prime}\right)$. For instance, $(a, u) \succ\left(a, u^{\prime}\right)$. There exists $b \in A$ such that $u(b)=u(a)$ and $u^{\prime}(b)=u^{\prime}(a)$. By $\sim \mathrm{PI},(b, u) \succ\left(b, u^{\prime}\right)$, which contradicts $\sim \mathrm{D}$ and $\sim \mathrm{C}$.

Let $u, u^{\prime} \in \mathcal{U}$ be such that for some $a \in X, u(a)=u^{\prime}(a)$, while for some $b, b^{\prime} \in A, b \gg b^{\prime}$ and $u(a)=u(b), u^{\prime}(a)=u^{\prime}\left(b^{\prime}\right)$. By $\sim \mathrm{D},(b, u) \succ\left(b^{\prime}, u^{\prime}\right)$, so that by $\sim \mathrm{PI},(a, u) \succ\left(a, u^{\prime}\right)$, contradicting $\sim \mathrm{IIU}$.

In this result, $\sim$ Continuity can be dropped if one replaces $\sim$ Weak Pareto by $\sim$ Strong Pareto and $\sim$ Dominance by its version involving large inequalities. 
The fairness approach is therefore singled out as a way to implement a reasonable compromise between Paretianism and the dominance idea. Observe, for instance, that the egalitarian-equivalent index given as an example in Section 6 satisfies $\sim$ Dominance on the thin set of all bundles proportional to $x_{0}$.

\section{Extension to opportunity sets}

The analysis of the Pareto-dominance dilemma can be extended to the case in which individual situations involve opportunity sets rather than bundles: $(A, u)$, where $A \subset X$.

Some pieces of notation have to be adapted. Now, $A \gg B$ means that for all $b \in B$, there is $a \in A$ such that $a \gg b$. Similarly, $A \geq B$ means that for all $b \in B$, there is $a \in A$ such that $a \geq b$. One has $A \geq B$ if and only if for all $u, v \in \mathcal{U}, u(A) \geq u(B)$, where $u(A)$ denotes the indirect utility obtained from $A$ when maximizing the utility function $u$. Let $\bar{X}$ denote the set of non-empty subsets of $X$, and $\bar{A}$ a subset of $\bar{X}$.

The dominance and Pareto axioms can be reformulated as follows.

Dominance on $\bar{A}: \forall A, B \in \bar{A}, \forall u, v \in \mathcal{U}$, if $A \gg B$, then $(a, u) \succ(b, v)$.

$\sim$ Weak Pareto on $\mathcal{V}: \forall A, B \in \bar{X}, \forall u \in \mathcal{V}$, if $u(A)>u(B)$, then $(A, u) \succ(B, u)$.

A set $\bar{A} \subset \bar{X}$ is said to be thin if for all $A, B \in \bar{A}$, either $A \geq B$ or $A \leq B$. Let

$$
B_{\varepsilon}(A)=\bigcup_{a \in A} B_{\varepsilon}(a)
$$

Theorem 7 Suppose $\bar{A}$ is such that for all $A \in \bar{A}, \varepsilon>0$, there is $B \subset B_{\varepsilon}(A)$ such that $B \gg A$, and if $A \gg\{0\}$, there is $C \subset B_{\varepsilon}(A)$ such that $C \ll A$.

(i) If $\succcurlyeq$ satisfies $\sim$ Dominance on $\bar{A}$ and $\sim$ Weak Pareto on $\mathcal{U}$, then $\bar{A}$ is thin.

(ii) If $\succcurlyeq$ satisfies $\sim$ Dominance on $X$ and $\sim$ Weak Pareto on $\mathcal{V}$, then $\mathcal{V}$ is thin.

(iii) If $\succcurlyeq$ satisfies $\sim$ Dominance on $\bar{A}$ and $\sim$ Weak Pareto on $\mathcal{V}$, then for all $A, B \in \bar{A}, u, v \in \mathcal{V}, u(A) \geq u(B)$ if and only $v(A) \geq v(B)$. 
Proof. The proof mimics that of Theorem 5. Suppose that there is $A, B \in \bar{A}$, $u, v \in \mathcal{V}$ such that $u(A) \geq u(B)$ and $v(A)<v(B)$. By continuity of the utility functions and the assumption on $\bar{A}$, one can find $A^{\prime} \in \bar{A}, A^{\prime} \gg A$ such that $u\left(A^{\prime}\right)>u(b)$ and $v\left(A^{\prime}\right)<v(B)$. By continuity of the utility functions and the assumption on $\bar{A}$ again, one can find $C, D \in \bar{A}$ such that $C \ll A^{\prime}, D \gg B$ and $u(C)>u(D)$. By $\sim$ Dominance on $\bar{A},(D, u) \succ(B, v)$ and $\left(A^{\prime}, v\right) \succ(C, u)$. By $\sim$ Weak Pareto on $\mathcal{V},(C, u) \succ(D, u)$ and $(B, v) \succ\left(A^{\prime}, v\right)$. One again obtains a cycle.

From the formal point of view, this extension to sets is immediate. One may, however, wonder if the axioms retain the same value in this setting. When dealing with sets, one might, in particular, think that the dominance idea becomes more compelling while the Pareto axiom is less so. As Basu (1994) notices, even the "Chicago school" defended the idea that two individuals are equally well-off if they choose in the same set, independently of their preferences:

$$
\forall A \subset X, \forall u, v \in \mathcal{U},(A, u) \sim(A, v) .
$$

While Basu shows that this idea conflicts with the Pareto criterion, we may note that this idea is the extension to sets of the above definition of perfectionnism, but seems less perfectionnist than in the bundle formulation.

It does not appear, however, that this kind of consideration makes the dominance idea immune to a problematic conflict with the Paretian idea of respecting individual preferences. After all, why should individuals not have a say in determining the shape of their opportunity sets, and why could their preferences be disregarded in this context? Truly enough, it may be less bad to give an individual a badly composed opportunity set than to give him a poor bundle, because the opportunity set still offers some choice. But it would obviously be even better to make the contents of the opportunity set reflect the orientation of the agent's preferences. In conclusion, the dilemma between dominance and Pareto, which can be settled in favor of Pareto in the bundle framework, should not be settled in favor of dominance when dealing with opportunity sets. A thin version of dominance seems to be warranted in this case as well.

A closely related analysis of the dominance idea is made in Pattanaik and $\mathrm{Xu}$ (2005). These authors do not introduce the Pareto axiom, but focus on a crucial consequence of the Pareto axiom when agents have different preferences, namely, that it may happen that a bundle or a set is better 
than another when they are given to agent $i$, while the reverse ranking would prevail for another agent $j$. They show that this occurrence is incompatible with the dominance axiom.

\section{Conclusion}

The theory of social choice has generally focused on the social aggregation problem, and has only indirectly touched upon the indexing problem, by looking at the consequences of properties of the index of interpersonal comparisons over the possibilities of social orderings, or by examining transfer axioms that added distributional preferences to comparative evaluations of individual situations. In this paper, a very simple formalism distinguishing the social ordering function and the index ordering has made it possible to formulate axioms bearing on the latter object and to study the construction of interpersonal comparisons on the basis of various kinds of information, namely, utility levels, indifference curves, dominance of bundles or sets.

Two well-known difficulties in the indexing problem have been analyzed in this light. First, the welfarism-perfectionnism dilemma has been shown to involve a third possibility, the fairness approach, which embraces Paretianism without relying on interpersonally comparable utility information. Second, the Pareto-dominance dilemma has been shown to be rather severe, although retaining the full force of the Pareto principle still makes it possible to apply the dominance principle in a restricted way. The two difficulties have been shown to be connected, and the compromise proposed for the latter singles out the fairness approach as a way to implement it. The fairness approach therefore emerges from this analysis as a way to combine the Paretian respect for individual preferences, the strict use of ordinal non-comparable information about preferences, and a maximal application of the dominance principle that is compatible with Pareto.

The literature contains numerous illustrations showing another feature of this approach that actually gives it its name, that is, the fact that it incorporates a variety of fairness principles which determine not only the method of social aggregation but also the precise way in which individual situations are measured and compared. In this way, the fairness approach fleshes out Rawls's rather enigmatic claim that the indexing problem "is not how to specify an accurate measure of some psychological or other attribute available only to science. Rather, it is a moral and practical problem." 


\section{References}

Arneson 1990, "Primary goods reconsidered", Noûs 24: 429-454.

Arrow K.J. 1963, Social Choice and Individual Values, New York: John Wiley (2nd revised ed.; 1st ed. 1951).

Arrow K.J. 1973, "Some ordinalist-utilitarian notes on Rawls's theory of justice", Journal of Philosophy 70: 245-263.

d'Aspremont C., L. Gevers 1977, "Equity and the informational basis of collective choice", Review of Economic Studies 44:199-210.

d'Aspremont C. 1985, "Axioms for Social Welfare Orderings", in L. Hurwicz, D. Schmeidler and H. Sonnenschein (Eds.), Social Goals and Social Organization, Cambridge: Cambridge University Press.

Basu K. 1994, "On interpersonal comparison and the concept of equality", in W. Eichhorn (Ed.), Models and Measurement of Welfare and Inequality, Berlin: Springer.

Bergson A. 1954, "On the Concept of Social Welfare", Quarterly Journal of Economics 68: 233-252.

Bordes G., M. Le Breton 1989, "Arrovian theorems with private alternatives domains and selfish individuals", Journal of Economic Theory 47: 257-281.

Broome J. 1991, Weighing Goods, Oxford: Blackwell.

Brun B.C., B. Tungodden 2004, "Non-welfaristic theories of justice: Is the intersection approach a solution to the indexing impasse?" Social Choice and Welfare 22: 4960.

Fleurbaey M. 2003, "On the Informational Basis of Social Choice", Social Choice and Welfare 21: 347-384.

Fleurbaey M. 2005, "Health, Wealth and Fairness", Journal of Public Economic Theory 7: 253-284.

Fleurbaey M., P.J. Hammond 2004, "Interpersonally Comparable Utility", in S. Barbera, P.J. Hammond and C. Seidl (Eds.), Handbook of Utility Theory, vol. 2: Extensions, Boston: Kluwer.

Fleurbaey M., F. Maniquet 2005, "Fair social orderings when agents have unequal skills", Social Choice and Welfare 24: 93-128.

Fleurbaey M., F. Maniquet 2006, "Fair income tax", Review of Economic Studies 73: 55-83.

Fleurbaey M., P. Mongin 2005, "The news of the death of welfare economics is greatly exaggerated", Social Choice and Welfare 25: 381-418.

Fleurbaey M., A. Trannoy 2003, "The impossibility of a Paretian Egalitarian", Social Choice and Welfare 21: 243-264 
Gibbard A. 1979, "Disparate goods and Rawls's difference principle: A social choice theoretic treatment", Theory and Decision 11: 267-288.

Kolm S.C. 1972, Justice et équité, Paris: Ed. du CNRS.

Pattanaik P., Y. Xu 2005, "Relativism, dominance, and standard of living comparisons based on functionings", mimeo.

Pazner E. 1979, "Equity, Nonfeasible Alternatives and Social Choice: A Reconsideration of the Concept of Social Welfare", in J.J. Laffont (Ed.), Aggregation and Revelation of Preferences, Amsterdam: North-Holland.

Pazner E., D. Schmeidler 1978, "Egalitarian Equivalent Allocations: A New Concept of Economic Equity", Quarterly Journal of Economics 92: 671-687. Rawls J. 1982, "Social unity and primary goods", in A.K. Sen et B. Williams (Eds.), Utilitarianism and Beyond, Paris: Maison des Sciences de l'Homme and Cambridge: Cambridge University Press.

Rawls J. 1993, Political Liberalism, Princeton: Columbia University Press.

Rawls J. 1999, A Theory of Justice, exp. ed., Oxford: Oxford University Press.

Robbins, L. 1932, An Essay on the Nature and Significance of Economics, London: McMillan (2nd revised ed., 1937).

Roemer J. E. 1996, Theories of Distributive Justice, Cambridge, Mass.: Harvard University Press.

Sen A.K. 1970, Collective Choice and Social Welfare, San Francisco: Holden Day.

Sen A.K. 1991, "On indexing primary goods and capabilities", mimeo. Sen A.K. 1992, Inequality Re-Examined, Oxford: Oxford University Press. Steinhaus H. 1948, "The Problem of Fair Division", Econometrica 16: 101104. 